OPEN

SUBJECT AREAS:

QUANTUM OPTICS

QUANTUM PHYSICS

PHYSICS

OPTICAL PHYSICS

Received

11 July 2012

Accepted

15 October 2012

Published

22 November 2012

Correspondence and requests for materials should be addressed to F.S. (fabio.sciarrino@ uniromal.it)

\section{Quantum interferometry with three-dimensional geometry}

\author{
Nicolò Spagnolo', Lorenzo Aparo', Chiara Vitelli ${ }^{1,2}$, Andrea Crespi ${ }^{3,4}$, Roberta Ramponi ${ }^{3,4}$, \\ Roberto Osellame ${ }^{3,4}$, Paolo Mataloni ${ }^{1,5}$ \& Fabio Sciarrino ${ }^{1,5}$
}

'Dipartimento di Fisica, Sapienza Università di Roma, Piazzale Aldo Moro 5, 1-00185 Roma, Italy, ${ }^{2}$ Center of Life NanoScience @ La Sapienza, Istituto Italiano di Tecnologia, Viale Regina Elena, 255, I-00185 Roma, Italy, ${ }^{3}$ Istituto di Fotonica e Nanotecnologie, Consiglio Nazionale delle Ricerche (IFN-CNR), Piazza Leonardo da Vinci 32, I-20133 Milano, Italy, ${ }^{4}$ Dipartimento di Fisica, Politecnico di Milano, Piazza Leonardo da Vinci 32, I-20133 Milano, Italy, 5 Istituto Nazionale di Ottica (INO-CNR), Largo E. Fermi 6, 1-50125 Firenze, Italy.

Quantum interferometry uses quantum resources to improve phase estimation with respect to classical methods. Here we propose and theoretically investigate a new quantum interferometric scheme based on three-dimensional waveguide devices. These can be implemented by femtosecond laser waveguide writing, recently adopted for quantum applications. In particular, multiarm interferometers include "tritter" and "quarter" as basic elements, corresponding to the generalization of a beam splitter to a 3- and 4-port splitter, respectively. By injecting Fock states in the input ports of such interferometers, fringe patterns characterized by nonclassical visibilities are expected. This enables outperforming the quantum Fisher information obtained with classical fields in phase estimation. We also discuss the possibility of achieving the simultaneous estimation of more than one optical phase. This approach is expected to open new perspectives to quantum enhanced sensing and metrology performed in integrated photonics.

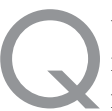

uantum metrology is one of the most fascinating frontiers of the science of measurement: the counterintuitive laws of quantum mechanics are exploited to maximize the amount of information extracted from an unknown sample, beating the limits imposed by classical physics. The low decoherence of photons, enabling the observation of quantum effects in an easier way, makes optical interferometry a promising candidate for demonstrating quantum enhanced sensitivity. The estimation of an optical phase $\phi$ through interferometric experiments is indeed an ubiquitous technique in physics, ranging from the investigation of fragile biological samples, such as tissues ${ }^{1}$ or blood proteins in aqueous buffer solution ${ }^{2}$, to gravitational wave measurements ${ }^{3,4}$. Whereas optical interferometry relying on classical interference is intrinsically a single-particle process, quantum advantages arise when quantum-correlated states of more than one particle are employed ${ }^{5}$, such as Greenberger-Horne-Zeilinger (GHZ) ${ }^{6}$ and $\mathrm{N} 00 \mathrm{~N}^{7}$ states. N00N states, in particular, allow to saturate the Heisenberg limit of sensitivity: the ultimate limit to the precision of a measurement imposed by the laws of physics.

Generally, the quantum advantage over classical approaches increases with the number of particles involved in the correlated probe state ${ }^{5}$. Two-photon N00N states can be produced deterministically simply by quantum interference of two identical photons on a balanced two-mode beam-splitter. However, two-mode N00N states with more than two particles are difficult to produce. Increasing the number of modes to more than two represents an interesting possibility to extend the concept of multiparticle interferometry, as pointed out by Greenberger et al. ${ }^{8}$. This requires multi-port devices, instead of simple two-mode beam-splitters, to build multiarm interferometers. Multi-port beam splitters can be potentially realized by properly combining several balanced two-port beam-splitters and phase shifters ${ }^{9,10}$ : such implementation has anyway tight requirements on interferometric stability, making its effective realization challenging with bulk optics. In fact, the few experimental realizations of such devices are reported on fiber-based ${ }^{11}$ or integrated-optics ${ }^{12}$ multi-mode devices; in both cases the characterization was performed with only two-photon states. Multi-photon interferometry is, indeed, still a widely unexplored field.

Times are mature to demonstrate multi-photon and multi-port devices. In fact, on the one hand in the last few years the efficiency of quantum multi-photon sources have dramatically improved leading to several experiments with up to 8 photons ${ }^{13}$. On the other hand, the advent of integrated quantum photonics have opened exciting perspectives for the realization of scalable, miniaturized and intrinsically stable optical setups ${ }^{14}$. In particular, the 


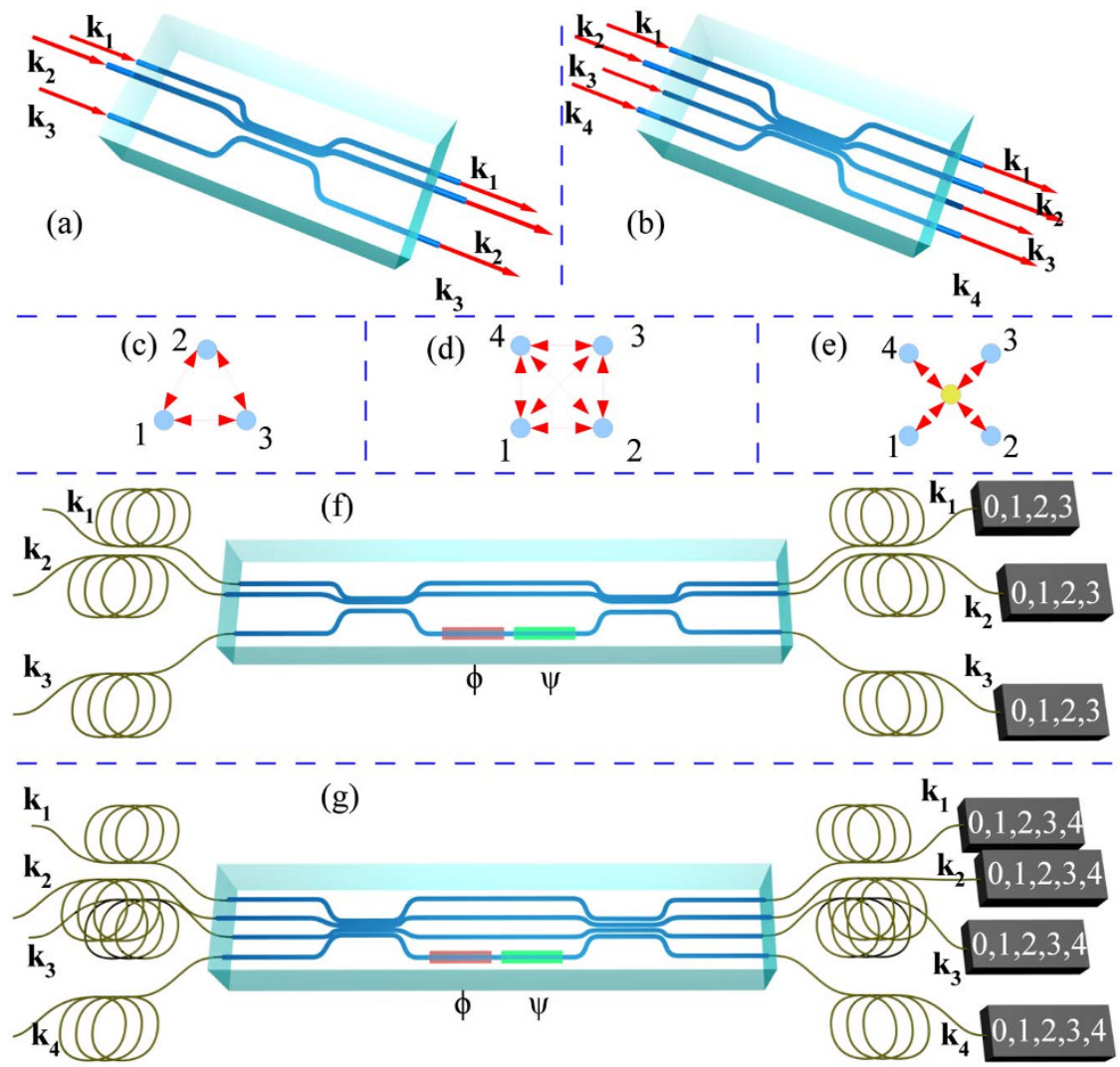

Figure $1 \mid$ (a) 3D structure of tritter. (b) 3-dimensional structure of quarter. (c) Geometry showing the direct coupling between the three modes of the tritter. (d) Geometry showing the direct coupling between the four modes of the quarter. (e) Indirect coupling between the four modes of the quarter by means of one ancillary mode. (f) 3-mode interferometer built by using two cascaded tritters. (g) 4-mode interferometer built by cascading two quarter devices. (f)-(g) $\phi$ : phase to be measured. $\psi$ : additional phase for adaptive phase estimation.

ultrafast laser-writing technique ${ }^{15-18}$ has proved to be a powerful tool for demonstrating new quantum integrated-optics devices, able to perform quantum logic operations ${ }^{19}$ as well as two-photon quantum walks $^{20,21}$. This technique exploits nonlinear absorption of focused femtosecond laser pulses to induce permanent and localized increase of the refractive index in transparent materials. Waveguides are directly fabricated in the material bulk by translating the sample at constant velocity along the desired path with unique three-dimensional (3D) capabilities.

In the field of quantum metrology, recent results have demonstrated the possibility of using two-mode path-entangled states in integrated structures for phase estimation below the Standard Quantum Limit (SQL) $)^{2,22-24}$. Combination of all the above elements would enable stepping into multi-photon/multi-port quantum metrology. However, the potentials of this approach have not yet been investigated theoretically.

In this work we introduce the concept of 3D multi-photon interferometry. First, we propose novel geometries for integrated multiarm interferometers based on three-port (tritter) and four-port (quarter) devices. Second, we theoretically study possible measurement protocols, based on the injection of multi-photon Fock states in this kind of multi-port devices, demonstrating relevant metrological advantages in phase-estimation tasks. Our results are not merely speculative since both the realization of such integrated multi-port devices $^{25}$ and the generation of multi-photon quantum states ${ }^{26}$ appear to be within reach of present state-of-the-art technology.

\section{Results}

Multi-arm interferometric schemes. A multi-arm interferometer can be realized by cascading two multi-port beam splitters. In particular, a three-arm interferometer is built by the combination of two tritters and a four arm interferometer by the combination of two quarters. In our approach the integrated-optics multi-port device is devised as a 3D multi-waveguide directional coupler [Fig. 1 (a)-(b)], a structure in which the waveguides are brought close together for a certain interaction length and coupled by evanescent field. The feasibility of such structures, in the case of three-ports (tritter), has already been demonstrated by femtosecond laser writing ${ }^{25,27}$, albeit characterization has only been performed in a classical framework. Note that in these compact multi-waveguide structures the interaction between the different arms happens simultaneously, without the decomposition into cascaded two-mode beam splitters. This is made possible by the $3 \mathrm{D}$ capabilities of femtosecond laser micromachining, thus relaxing the strict requirements on path-length control of alternative approaches.

The symmetric configuration of a tritter can be easily obtained by adopting a triangular geometry, as shown in Fig. 1 (c). In this configuration it is possible to obtain equal coupling coefficients, so that a single photon entering in one input port has the same probability of exiting from one of the three output ports. The symmetric configuration of a quarter can be achieved by adopting two possible solutions. In the first one, the four modes are directly coupled, and the symmetric condition can be obtained by appropriately tuning the interaction length [Fig. 1 (d)]. Alternatively, an indirect geometry may be exploited by coupling waveguides 1-4 through an ancillary mode, as shown in Fig. 1 (e), and without any direct coupling between them.

Output states. We start by considering the action of a single tritter and of a single quarter. The action of these devices on an input state $|\psi\rangle$ is expressed by a unitary matrix $\mathcal{U}^{(I I I)}\left[\mathcal{U}^{(I V)}\right]$, which maps the input field operators $a_{i}^{\dagger}$ to the output field operators $b_{i}^{\dagger}$ according to 

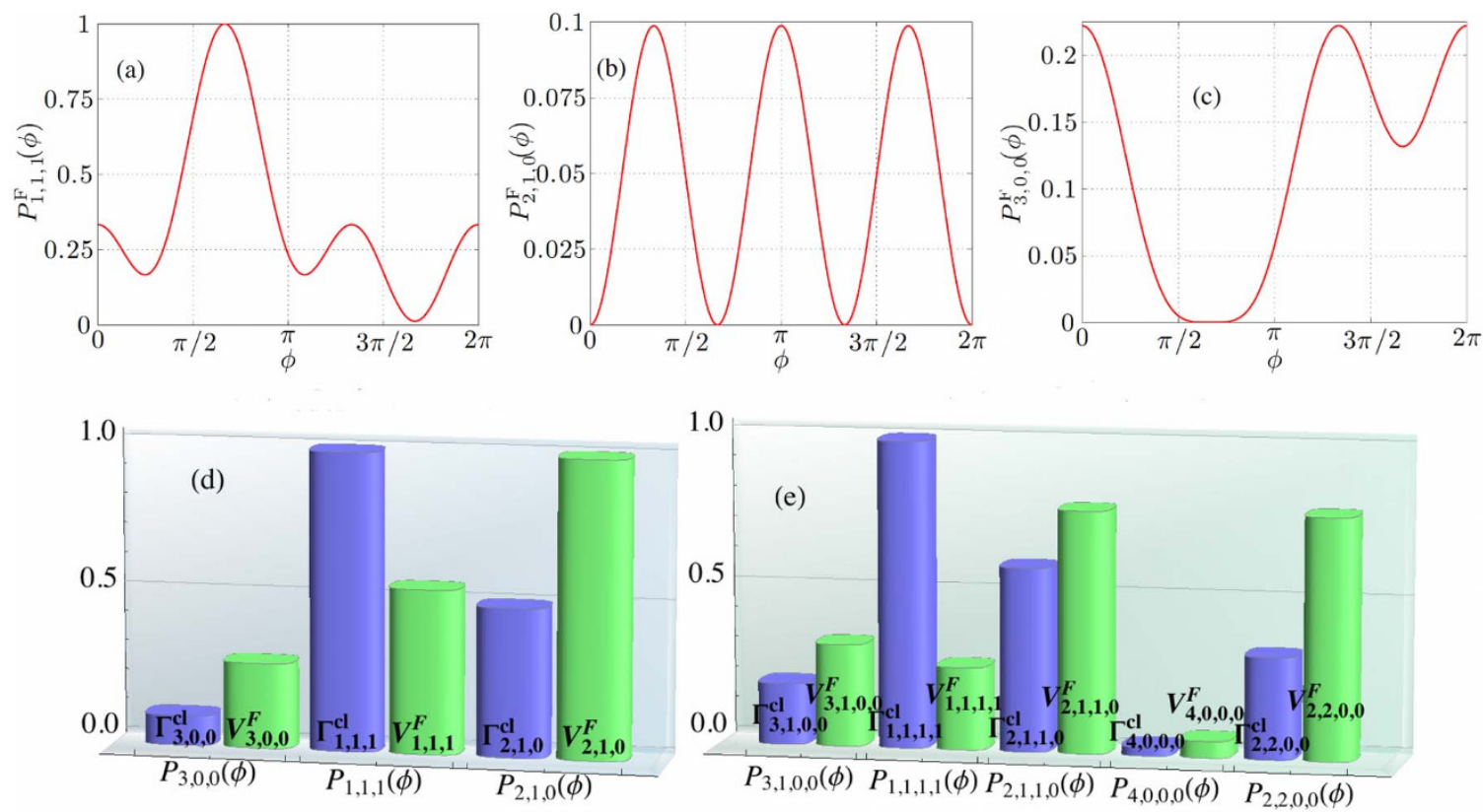

Figure $2 \mid$ (a)-(c) Output fringe patterns $P_{m, n, q}^{F}$ of the 3-modes interferometer fed with a $|1,1,1\rangle$ input state. (d) Diagram of the $N$-fold visibilities $V_{m, n, q}^{F}$ of the output fringe patterns $P_{m, n, q}^{F}$ for a $N=3$ interferometer with a $|1,1,1\rangle$ input state, compared with the classical bound $\Gamma_{m, n, q^{*}}^{c l}$ (e) Corresponding diagram for the 4-modes case.

$b_{i}^{\dagger}=\sum_{i, j} \mathcal{U}_{i j}^{(k)} a_{j}^{\dagger}$, with $k=I I I, I V^{9,10,28}$ (see Supplementary Material). Let's consider the Fock state $|1,1,1\rangle$, where $|i, j, l\rangle=|i\rangle_{\mathrm{k}_{1}}|j\rangle_{\mathrm{k}_{2}}|l\rangle_{\mathrm{k}_{3}}$ is the input state in the tritter. By applying $\mathcal{U}^{(I I I)}$ we obtain the output state:

$$
|1,1,1\rangle \rightarrow c_{\{1,1,1\}}|1,1,1\rangle+c_{\{3,0,0\}}|\{3,0,0\}\rangle .
$$

Here $c_{1,1,1}=-e^{i 2 \pi / 3} / \sqrt{3}, c_{\{3,0,0\}}=e^{i 4 \pi / 3} \sqrt{2 / 3}$, and $|\{i, j, l\}\rangle$ is the symmetric superposition of three-photon states where $(i, j, l)$ photons exit in the three output ports. A similar result is obtained for a quarter fed with a $|1,1,1,1\rangle=|1\rangle_{\mathrm{k}_{1}}|1\rangle_{\mathrm{k}_{2}}|1\rangle_{\mathrm{k}_{3}}|1\rangle_{\mathrm{k}_{4}}$ state:

$$
\begin{aligned}
& |1,1,1,1\rangle \rightarrow c_{\{1,1,1,1\}}|1,1,1,1\rangle+ \\
& c_{\{2,2,0,0\}}|\{2,2,0,0\}\rangle+c_{\{4,0,0,0\}}|\{4,0,0,0\}\rangle,
\end{aligned}
$$

where $c_{1,1,1,1}=1 / 2, c_{\{2,2,0,0\}}=\sqrt{6} / 4, c_{\{4,0,0,0\}}=-\sqrt{6} / 4$. In both cases, we observe that some terms of the output states are suppressed due to quantum interference. They correspond in particular to the contributions $\{2,1,0\}$ in the tritter case, $\{3,1,0$, $0\}$ and $\{2,1,0,0\}$ in the quarter one. This feature is a $\mathrm{N}$-mode analogue of the Hong-Ou-Mandel bosonic coalescence effect ${ }^{28-30}$. The two devices can be exploited to generate maximally-entangled $\mathrm{N} 00 \mathrm{~N}$ states in a post-selected configuration ${ }^{31}$.

$\mathrm{N}$-modes interferometry. The $3 \mathrm{D}, \mathrm{N}$-port structure of tritter and of quarter devices can be exploited to implement an integrated $\mathrm{N}$-modes interferometer. This can be realized by a chain of two subsequent multiport beam-splitter, leading to a generalized MachZehnder structure [see Fig. 1 (f)-(g)]. The phase shifting could be introduced for instance by adopting a microfluidic channel as reported in Ref. 2,32. Let us now consider the 3-modes system obtained with two tritter devices, and the action of a relative phase shift $\phi$ in the optical mode $\mathbf{k}_{3}$ inside the interferometer, which is described by the operator $\mathcal{U}_{\phi}=\exp \left(-\imath n_{3} \phi\right)$, being $n_{3}$ the photon number operator for mode $\mathbf{k}_{3}$. The output probability distributions $P_{m, n, q}(\phi)$ corresponding to the detection of $(m, n, q)$ photons in the three output ports, are obtained by the overall evolution of the interferometer $\mathcal{U}^{(I I I)} \mathcal{U}_{\phi} \mathcal{U}^{(I I I)}$ acting on the input state. Let us consider again an input Fock state $|1,1,1\rangle$; the obtained fringe patterns $P_{m, n, q}^{\mathrm{F}}(\phi)$, symmetric for an index exchange $(m, n, q)$, are reported in Figs. 2 (a)-(c). We observe the presence of interferometric patterns presenting the sum of different harmonics up to $\cos (3 \phi)$. Furthermore we note that the $P_{2,1,0}^{\mathrm{F}}(\phi)$ term presents a sub-Rayleigh $\lambda / 3$ behavior with a unitary visibility. These results suggest that the output state of the interferometer, for a $|1,1,1\rangle$ input state, presents nonclassical features. Similar results can be obtained when a 4-modes interferometer is fed with a $|1,1,1,1\rangle$ input state [Fig. 1 (g)] (see Supplementary Material).

Nonclassicality criterion for sub-Rayleigh fringe patterns. In order to formally address the nonclassicality of this setup we have extended the criterion proposed by Afek et al. in Ref. [33] from a 2-modes to a 3 -modes interferometer. This criterion sets an upper bound $\Gamma_{m, n, q}^{\mathrm{cl}}$ for classical states on the $N$-fold visibilites of the Fourier expansion of the fringe pattern distributions $P_{m, n, q}(\phi)=\sum_{k=0}^{\infty} A_{k} \cos \left(k \phi-\delta_{k}\right)$, where $N=m+n+q$. The visibilities are defined as the ratio between the Fourier coefficient $A_{m, n, q}$ of the term oscillating as $N \phi$ and the constant coefficient $A_{0}$, i.e. $V_{m, n, q}=\left|A_{m, n, q} / A_{0}\right|$. The classical bound for $V_{m, n, q}$ is obtained by calculating the probability distribution of having $(m, n, q)$ photons in the output modes $(1,2$, 3) respectively, by feeding the interferometer with a classical coherent state: $\left|\alpha_{1}, \alpha_{2}, \alpha_{3}\right\rangle$, where $\alpha_{i}=\left|\alpha_{i}\right| e^{l \theta_{i}}$. Then, the $N$-fold visibility $V_{m, n, q}$ is maximized with respect to $\left|\alpha_{i}\right|$ and $\theta_{i}$, leading to $\max V_{m, n, q}=\Gamma_{m, n, q}^{\mathrm{cl}}$. In general, it can be shown that for any classical state $V_{m, n, q} \leq \Gamma_{m, n, q}^{\mathrm{cl}}$, since any classical state can be expanded in the coherent states basis according to $\rho_{C}=\int d^{2} \alpha P(\alpha)|\alpha\rangle\langle\alpha|$ with a well behaved $P(\alpha)$. The corresponding $N$-fold visibilities $V_{m, n, q}^{\mathrm{F}}$ for the input Fock state $|1,1,1\rangle$ are obtained from the probability distributions $P_{m, n, q}^{\mathrm{F}}$ shown in Figs. 2 (a)-(c), leading to higher visibilities than the classical limits, except for the $P_{1,1,1}^{F}$ case [Fig. 2 (d)]. The same criterion can be extended to a $\mathrm{N}$-modes interferometer. We then repeated the same analysis for the $N=4$ case when the interferometer is built by two subsequent quarter devices [Fig. 1 (g)], showing the presence of nonclassical behaviors in the fringe patterns [see Fig. $2(\mathrm{e})$ ].

Phase estimation. The present interferometric configuration can be adopted to perform a phase estimation protocol. In this context, the 

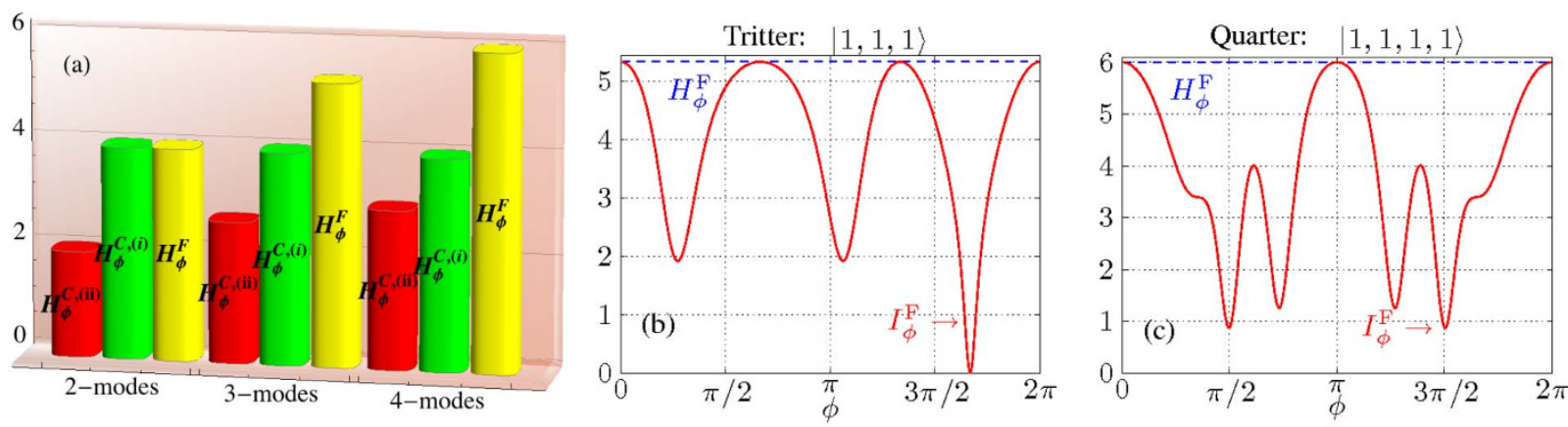

Figure $3 \mid$ (a) Comparison of the QFIs for the $N=2, N=3, N=4$ interferometers fed with a Fock state $H_{\phi}^{\mathrm{F}}$ a coherent state with an external phase reference beam $H_{\phi}^{\mathrm{C},(\mathrm{i})}$, and a coherent state without an external phase reference beam $H_{\phi}^{\mathrm{C} \text {,(ii) }}$. (b)-(c) QFI $H_{\phi}^{\mathrm{F}}$ and FI $I_{\phi}^{\mathrm{F}}$ for (b) a 3-modes interferometer with a $|1,1,1\rangle$ input state, and (c) for a 4-modes interferometer with an input $|1,1,1,1\rangle$ state. The optimal working points are obtained when $I_{\phi}^{\mathrm{F}}=H_{\phi}^{\mathrm{F}}$.

aim is to measure an unknown phase shift $\phi$ introduced in an interferometer with the best possible precision by probing the system with a $\mathrm{N}$-photon state, and by measuring the resulting output state. The classical limit is provided by the SQL, which sets a lower bound to the minimum uncertainty $\delta \phi_{\mathrm{SQL}} \geq 1 / \sqrt{M N}$ which can be obtained on $\phi$ by exploiting classical $N$-photon states on two modes and $M$ repeated measurements ${ }^{34}$. Recently, it has been shown that the adoption of quantum states can lead to a better scaling with $N$, setting the ultimate precision to $\delta \phi_{\mathrm{HL}} \geq 1 /(\sqrt{M} N)$, corresponding to the Heisenberg limit ${ }^{5,35,36}$. Hereafter, we show that the present integrated technology can lead to a sub-SQL performance in the estimation of an optical phase, exploiting multi-mode interferometry.

Quantum fisher information. In order to characterize the present 3modes interferometer fed with a $|1,1,1\rangle$ input state, and analogously the 4-modes case, we need to determine the quantum Fisher information (QFI) $H_{\phi}^{\mathrm{F}}$ of the output state ${ }^{37,38}$. This quantity sets the maximum amount of information which can be extracted on the phase $\phi$ from a state $\varrho_{\phi}$ according to the quantum Cramér-Rao (QCR) bound: $\delta \phi \geq\left(M H_{\phi}\right)^{-1 / 2} 37$. The classical limit is provided by the quantum Fisher information $H_{\phi}^{\mathrm{C}}$ when a coherent state $\left|\alpha_{1}, \alpha_{2}, \alpha_{3}\right\rangle$ is injected into the interferometer. Note that the comparison between the performances achievable with an input coherent state and an input $|1,1,1\rangle$ Fock state must be performed for the same number of photons impinging onto the phase shifter. Furthermore, for a coherent state input two different cases can be identified. (i) If an external reference beam, providing an absolute reference frame for the optical phase, is available at the measurement stage, the QFI is calculated for a pure $\left|\alpha_{1}, \alpha_{2}, \alpha_{3}\right\rangle$ input state: $H_{\phi}^{\mathrm{C},(i)}$. (ii) If no reference frame is available at the measurement stage (such as for photoncounting detection), one needs to average the input state on a random phase shift $\theta$ common to all input modes leading to $H_{\phi}^{\mathrm{C},(i i)}$. In absence of an external reference beam, an input state $\varrho$ has to be replaced with the phase-averaged state $\varrho^{\prime}=(2 \pi)^{-1} \int_{0}^{2 \pi}$ $d \theta \mathcal{U}_{\theta}^{1} \mathcal{U}_{\theta}^{2} \mathcal{U}_{\theta}^{3} \varrho \mathcal{U}_{\theta}^{1 \dagger} \mathcal{U}_{\theta}^{2 \dagger} \mathcal{U}_{\theta}^{3 \dagger}$, where $\mathcal{U}_{\theta}^{i}$ is the phase shift operator for mode $\mathbf{k}_{i}$. The two conditions (i) and (ii) are equivalent for the $\mid 1,1$, 1) probe, since this state has a fixed number of photons ${ }^{39}$. We then evaluated the three quantities $H_{\phi}^{\mathrm{C},(i)}, H_{\phi}^{\mathrm{C},(i i)}, H_{\phi}^{\mathrm{F}}$, and obtained that the adoption of $\mathrm{a}|1,1,1\rangle$ probe state leads to quantum improved performances. The same result is found for the 4-modes case, as shown in Fig. 3 (a). We note that, while $H_{\phi}^{\mathrm{C},(i)}$ is fixed, the QFI achievable with a Fock state input increases with the number of modes, leading progressively to a greater advantage in phase estimation protocols. Furthermore, no post-selection is needed to generate the required probe state $^{40}$.

Achieving the optimal bound. We now show that the QCR bound provided by $H_{\phi}^{\mathrm{F}}$ can be achieved by adopting a feasible and practical choice of the measurement setup, consisting in a photon-counting apparatus recording the number of output photons on each mode. The detection apparatus can be implemented by splitting each output mode in three parts by means of a chain of beam-splitters, and by placing a single-photon detector on each part. The occurrence of 1,2 or 3 simultaneous clicks of the detectors on the same mode corresponds to the detection of 1, 2, 3 photons. For a fixed choice of the measurement setup, the amount of information which can be extracted on $\phi$ is provided by the Cramér-Rao (CR) bound $\delta \phi \geq$ $\left(M I_{\phi}\right)^{-1 / 237}$, where $I_{\phi}$ is the Fisher information of the output probability distribution of the measurement outcomes. The results for $I_{\phi}^{\mathrm{F}}$ with the $|1,1,1\rangle$ input state and photon-counting measurements are reported in Fig. 3 (b). By comparing the trend of $I_{\phi}^{\mathrm{F}}$ with the corresponding QFI $H_{\phi}^{\mathrm{F}}$, we observe that the ultimate precision given by the QCR bound can be achieved with this choice of the measurement apparatus for $\phi=0,2 \pi / 3,4 \pi / 3$. An analogous result is found for the $N=4$ case, where the optimal working points are now $\phi=0, \pi$ [Fig. 3 (c)].

Adaptive protocol. The obtained $\phi$-dependence of the Fisher information $I_{\phi}$ suggests that an adaptive protocol ${ }^{41}$ is necessary to obtain optimal performances in the full phase range, that is, to saturate the QCR bound for all values of $\phi$. To this purpose, we consider the adoption of a three-step adaptive strategy where the first two steps of the protocol are performed to obtain a rough estimate $\phi_{\mathrm{r}}$ of the phase $\phi$ (more details on the protocol can be found in the Supplementary information). The amount of measurements performed in these steps is a small fraction of the overall resources $M$, namely $M_{1}=M_{2}=\sqrt{M}$. In the first step, a rough estimate of the phase is obtained up to a two-fold degeneracy due to the symmetry of the interferometric fringes $(\phi, 4 \pi / 3-$ $\phi)$. The second step is exploited to remove this degeneracy for the estimate $\phi_{\text {est. }}$. Finally, the third step consisting of $M_{3}=M-M_{1}-M_{2}$ measurements is performed by sending the $|1,1,1\rangle$ input state, and the system is tuned to operate in the optimal regime $(\phi+\psi \simeq 2 \pi / 3)$ by means of an additional phase shift $\psi$. At each step of the protocol, the measurement outcomes are analyzed by a Bayesian approach and assuming no a-priori knowledge on $\phi^{42}$. The results of the numerical simulation for $M=10^{5}$ are reported in Fig. 4, together with the results for a numerical simulation of a one step non-adaptive strategy. We observe that in the latter case, the uncertainty $\delta \phi$ associated to the estimation process resembles closely the CR bound provided by the Fisher information $I_{\phi}^{\mathrm{F}}$. A better result is obtained with the adaptive strategy, showing that the quantum Fisher information is achieved, leading to sub-SQL performances in the full phase interval. The choice of the Bayesian approach leads to an unbiased estimation process, i.e., the estimated phase $\phi_{\text {est }}$ converges to the true value $\phi$ (see inset of Fig. 4), and the error on the estimation process can be directly retrieved from the output distribution for $\phi^{43}$. 


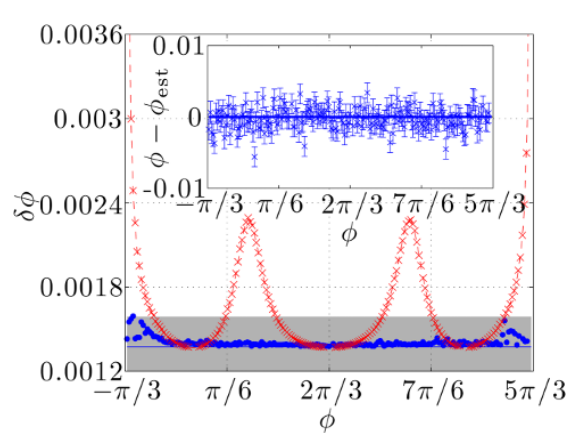

Figure $4 \mid$ Results for the phase estimation error $\delta \phi$ of a numerical simulation with $M=10^{5}$ repeated measurements with the three modes interferometer of Fig. 1 (f). Blue circular points: $\delta \phi$ for the adaptive protocol as a function of $\phi$. Blue solid line: QCR bound given by $H_{\phi}^{\mathrm{F}}$. Red cross points: $\delta \phi$ for the non-adaptive protocol. Red dashed line: Fisher information $I_{\phi}^{\mathrm{F}}$, providing the bound for the non-adaptive strategy. Shaded region corresponds to sub-SQL performances achievable with quantum resources. Inset: plot of the difference between the true value $\phi$ and the estimated value $\phi_{\text {est }}$ obtained with the adaptive protocol.

Two parameter phase estimation. Let us now consider a different scenario. The 3-modes interferometer built with two cascaded tritters proposed in this paper may be adopted to perform a two parameters estimation process, consisting of the simultaneous measurement of two optical phases. In this case, the reference $\phi_{\text {ref }}$ is provided by the optical mode $\mathbf{k}_{1}$, and the phases to be measured, $\phi_{2}$ and $\phi_{3}$, correspond respectively to the optical modes $\mathbf{k}_{2}$ and $\mathbf{k}_{3}$ [see Fig. 5 (a)]. In order to evaluate the maximum precision achievable in the two parameter problem, it is necessary to extend the concept of quantum Fisher information to the multiparameter case ${ }^{44}$. Indeed, it is possible to define a quantum Fisher information matrix (QFIM) $\mathbf{H}_{\mu v}$ corresponding to the set of parameter $\lambda=\left(\lambda_{1}, \ldots, \lambda_{n}\right)$, which in the pure state case $\left|\psi_{\lambda}\right\rangle=e^{-l \sum_{\mu} G_{\mu} \lambda_{\mu}}\left|\psi_{0}\right\rangle$ is defined in terms of the set of generators $\mathrm{G}=\left(G_{1}, \ldots, G_{n}\right)$ for the parameters $\lambda$. The error on the single parameter $\lambda_{\mu}$ for a fixed value of the other parameters $\lambda_{v}$, with $v \neq \mu$, is bounded by the inequality: $\delta \lambda_{\mu} \geq\left[\left(\mathrm{H}^{-1}\right)_{\mu \mu} / M\right]^{1 / 2}$. When performing the simultaneous estimation of the set of parameters $\lambda$, the sum of the variances is bounded by the multiparameter Cramér-Rao inequality: $\sum_{\mu} \operatorname{Var}\left(\lambda_{\mu}\right) \geq \operatorname{Tr}\left[\mathbf{H}^{-1}\right] / M$.

We now consider as input state a coherent state $|\alpha\rangle$ with $\langle n\rangle=3$, which defines the standard quantum limit for a $N=3$ photon probe. As for the single parameter scenario, it is necessary to evaluate the quantum Fisher information matrices both (i) in presence of an external phase reference $\mathrm{H}_{\phi_{2}, \phi_{3}}^{C,(i)}$ and (ii) in absence of an external phase reference $\mathrm{H}_{\phi_{2}, \phi_{3}}^{C,(i i)}$. The corresponding bounds for the sensitivities after $M$ measurements in the two cases are given by $\delta \phi_{\mu}^{C,(i)} \geq\left(M \tilde{H}_{\phi_{\mu}}^{C,(i)}\right)^{-1 / 2}$ and $\delta \phi_{\mu}^{C,(i i)} \geq\left(M \tilde{H}_{\phi_{\mu}}^{C,(i i)}\right)^{-1 / 2}$. When a three photon state $|1,1,1\rangle$ is injected into the interferometer, the bounds for the parameters $\phi_{2}$ and $\phi_{3}$ are the same for both cases (i) and (ii) leading to $\delta \phi_{\mu}^{F} \geq\left(M \tilde{H}_{\phi_{\mu}}^{F}\right)^{-1 / 2}$. The same analysis may be performed in the case of a 4-modes interferometer, fed with a coherent state of $\langle n\rangle=4$ photons or with a Fock state $|1,1,1,1\rangle$, where the two parameters are now the phases $\phi_{3}$ and $\phi_{4}$ on modes $\mathbf{k}_{3}$ and $\mathbf{k}_{4}$ [see Fig. 5 (b)]. The results are shown in Fig. 5 (c), showing that in absence of a phase reference the adoption of Fock probe states can lead to quantum enhanced performances in the measurement of two optical phases. Furthermore, in full analogy with the one parameter case, a greater advantange with respect to the classical strategies may be progressively achieved by increasing the number of modes.

In the single parameter case, the quantum Cramér-Rao bound can always be asymptotically achieved ${ }^{41}$ performing a suitable measurement and choosing the right estimator. On the contrary, in the multiparameter case the bound for the statistical errors defined by the quantum Fisher information matrix is not in general achievable $e^{45,46}$. This depends on the fact that the optimal measurements for the individual parameters may not be compatible observables. A necessary condition for the achievability of the multiparameter quantum Cramér-Rao bound is then given by the weak commutativity condition: $\operatorname{Tr}\left[\rho_{\lambda}\left[L_{\mu}, L_{v}\right]\right]=0$. Here, the $L_{\mu}$ operators are the symmetric logarithmic derivatives (SLD), which define the optimal measurement and estimators for the individual parameters ${ }^{47}$. In our case, it can be shown that (see Supplementary Material) for the 3-mode interferometer injected by the $|1,1,1\rangle$ input state the operators $\left\{L_{2}, L_{3}\right\}$ for $\left\{\phi_{2}, \phi_{3}\right\}$ commute, thus satisfying the necessary condition to achieve the quantum Cramér-Rao bound. The same result holds for the 4-mode case. The next step to be investigated is the identification of suitable measurements and estimators.

\section{Discussions}

The analysis performed in this work represents the first step in the investigation of integrated quantum technology in view of the realization of multiport optical beam splitters enabling novel multiphoton sensing schemes based on 3D interferometers. We investigated the adoption of this technology to perform phase estimation protocols leading to quantum-enhanced performances. We provided and simulated a full protocol for sub-SQL phase measurements, by exploiting Fock input states and photon-counting detection, thus not requiring any post-selection for the generation of the probe state. We also discussed the application of the same multimode structure for multiparameter estimation purposes. The present technology is expected to lead to the development of new phase estimation protocols able to reach Heisenberg-limited performances ${ }^{31}$ and to open a new scenario for the simultaneous measurement of more than one optical phase. Indeed, the present approach can be adopted as an (a)

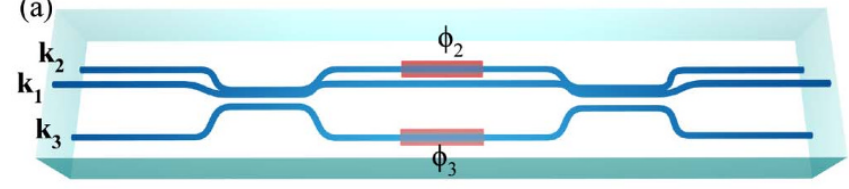

(b)

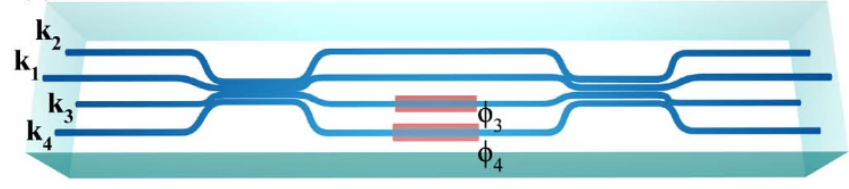

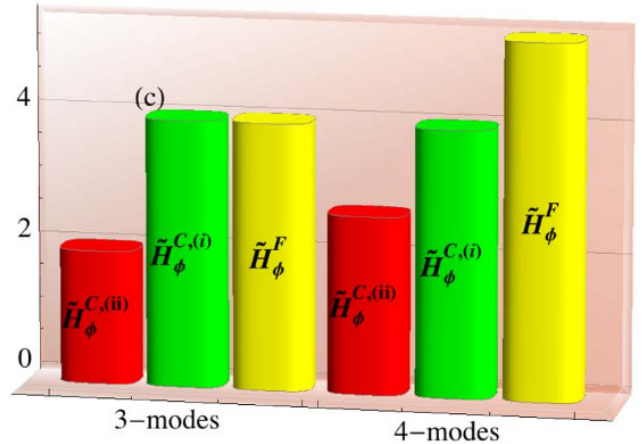

Figure 5 $\mid$ (a)-(b) Schemes for two parameters phase estimation with the 3-and 4-modes integrated interferometers. (c) Comparison between the effective quantum Fisher informations $\left(\tilde{H}_{\phi \mu}^{C,(i)}, \tilde{H}_{\phi \mu}^{C,(i i)}, \tilde{H}_{\phi \mu}^{F}\right)$ for the two-parameters problem when the interferometer is fed with Fock states or coherent states. 
accessible test bench to investigate theoretically and experimentally the still unexplored scenario of multiparameter estimation. Further perspectives may lead to the application of this multiport splitters in other contexts, such as quantum simulations ${ }^{48}$, linear optical computing $^{49}$ and nonlocality tests ${ }^{50}$.

1. Nasr, M. B. et al. Quantum optical coherence tomography of a biological sample. Optics Communications 282, 11541159 (2009).

2. Crespi, A. et al. Measuring protein concentration with entangled photons. Appl. Phys. Lett. 100, 233704 (2012).

3. Goda, K. et al. A quantum-enhanced prototype gravitational-wave detector. Nature Physics 4, 472 (2008).

4. The LIGO Scientific Collaboration. A gravitational wave observatory operating beyond the quantum shot-noise limit: Squeezed light in application. Nature Physics 7, 962 (2011)

5. Giovannetti, V., Lloyd, S. \& Maccone, L. Quantum-enhanced measurements: Beating the standard quantum limit. Science 306, 1330 (2004).

6. Bouwmeester, D., Pan, J.-W., Daniell, M., Weinfurter, H. \& Zeilinger, A. Observation of the three-photon greenberger-horne-zeilinger entanglement. Phys. Rev. Lett. 82, 1345 (1999).

7. Boto, A. N. et al. Quantum interferometric optical lithography: Exploiting entanglement to beat the diffraction limit. Phys. Rev. Lett. 85, 2733 (2000).

8. Greenberger, D. M., Home, M. A. \& Zeilinger, A. Similarities and difference between two-particle and three-particle interference. Fortschr. Phys. 48, 243 (2000).

9. Zukowski, M., Zeilinger, A. \& Horne, M. A. Realizable higher-dimensional twoparticle entanglements via multiport beam splitters. Phys. Rev. A 55, 2564 (1997).

10. Campos, R. A. Three-photon hong-ou-mandel interference at a multiport mixer. Phys. Rev. A 62, 013809 (2000)

11. Weihs, G., Reck, M., Weinfurter, H. \& Zeilingerr, A. Two-photon interference in optical fiber multiports. Phys. Rev. A 54, 893 (1996).

12. Peruzzo, A., Laing, A., Politi, A., Rudolph, T. \& O’Brien, J. L. Multimode quantum interference of photons in multiport integrated devices. Nature Communications 2, 244 (2011).

13. Yao, X. C. et al. Experimental demonstration of topological error correction. Nature (London) 482, 489 (2012).

14. Politi, A., Cryan, M. J., Rarity, J. G., Yu, S. \& O’Brien, J. L. Silica-on-silicon waveguide quantum circuits. Science 320, 646 (2008).

15. Gattass, R. R. \& Mazur, E. Femtosecond laser micromachining in transparent materials. Nature Photonics 2, 219 (2008).

16. Della Valle, G., Osellame, R. \& Laporta, P. Micromachining of photonic devices by femtosecond laser pulses. J. Opt. A: Pure Appl. Opt. 11, 013001 (2009).

17. Osellame, R., Hoekstra, H. J. W. M., Cerullo, G. \& Pollnau, M. Femtosecond laser microstructuring: an enabling tool for optofluidic lab-on-chips. Laser Phot. Rev. 5 , $442(2011)$

18. Nolte, S., Will, M., Burghoff, J. \& Tuennermann, A. Femtosecond waveguide writing: a new avenue to three-dimensional integrated optics. Applied Physics A: Materials Science \& Processing 77, 109 (2003).

19. Crespi, A. et al. Integrated photonics quantum gates for polarization qubits. Nature Communications 2, 566 (2011).

20. Owens, J. O. et al. Two-photon quantum walks in an elliptical direct-write waveguide array. New Journal of Physics 13 (2011).

21. Sansoni, L. et al. Two-particle bosonic-fermionic quantum walk via integrated photonics. Phys. Rev. Lett. 108, 010502 (2012).

22. Smith, B. J., D. Kundys, Thomas-Peter, N., Smith, P. G. R. \& Walmsley, I. A. Phase-controlled integrated photonic quantum circuits. Optics Express 17, 13516 (2009).

23. Matthews, J. C. F., Politi, A., Bonneau, D. \& O’Brien, J. L. Heralding two-photon and four-photon path entanglement on a chip. Phys. Rev. Lett. 107, 163602 (2011).

24. Shadbolt, P. J. et al. Generating, manipulating and measuring entanglement and mixture with a reconfigurable photonic circuit. Nature Photonics 6, 45 (2011).

25. Kowalevicz, A. M. et al. Three-dimensional photonic devices fabricated in glass by use of a femtosecond laser oscillator. Optics Letters 30, 1060 (2005).

26. Metcalf, B. J. et al. Multi-photon quantum interference in a multi-port integrated photonic device. arXiv:1208.4575 (2012).

27. Suzuki, K., Sharma, V., Fujimoto, J. G., Ippen, E. P. \& Nasu, Y. Characterization of symmetric [3 $\mathrm{x} 3]$ directional couplers fabricated by direct writing with a femtosecond laser oscillator. Opt. Express 14, 2335 (2006).
28. Tichy, M. C., Tiersch, M., de Melo, F., Mintert, F. \& Buchleitner, A. Zerotransmission law for multiport beam splitters. Phys. Rev. Lett. 104, 220405 (2010).

29. Hong, C. K., Ou, Z. \& Mandel, L. Measurement of subpicosecond time intervals between two photons by interference. Phys. Rev. Lett. 59, 2044 (1987).

30. Ou, Z. Y., Rhee, J.-K. \&Wang, L. J. Observation of four-photon interference with a beam splitter by pulsed parametric down-conversion. Phys. Rev. Lett. 83, 959-962 (1999).

31. Pryde, G. J. \& White, A. G. Creation of maximally entangled photon-number states using optical fiber multiports. Phys. Rev. A 68, 052315 (2003).

32. Crespi, A. et al. Three-dimensional mach-zehnder interferometer in a microfluidic chip for spatially-resolved label-free detection. Lab Chip 10, 1167 (2010).

33. Afek, I., Ambar, O. \& Silberberg, Y. Classical bound for mach-zehnder superresolution. Phys. Rev. Lett. 104, 123602 (2010).

34. Ou, Z. Y. Fundamental quantum limit in precision phase measurement. Phys. Rev. A 55, 2598 (1997).

35. Giovannetti, V., Lloyd, S. \& Maccone, L. Quantum metrology. Phys. Rev. Lett. 96, 010401 (2006).

36. Giovannetti, V., Lloyd, S. \& Maccone, L. Advances in quantum metrology. Nature Photonics 5, 222 (2011).

37. Helstrom, C. W. Quantum Detection and Estimation Theory (Academic Press, 1976).

38. Braunstein, S. L., Caves, C. M. \& Milburn, G. J. Generalized uncertainty relations: Theory, examples, and lorentz invariance. Annals of Physics 247, 135 (1996).

39. Jarzyna, M. \& Demkowicz-Dobrzanski, R. Quantum inteferometry with and without an external phase reference. Phys. Rev. A 85, 011801 (2012).

40. Resch, K. J. et al. Time-reversal and super-resolving phase measurements. Phys. Rev. Lett. 98, 223601 (2007).

41. Nagaoka, H. An asymptotic efficient estimator for a one-dimensional parametric model of quantum statistical operators. Proc. Int. Symp. on Inform. 198 (1988).

42. Pezzé, L., Smerzi, A., Khoury, G., Hodelin, J. F. \& Bouwmeester, D. Phase detection at the quantum limit with multiphoton mach-zehnder interferometry. Phys. Rev. Lett. 99, 223602 (2007).

43. Krischek, R. et al. Useful multiparticle entanglement and sub-shot-noise sensitivity in experimental phase estimation. Phys. Rev. Lett. 107, 080504 (2011).

44. Paris, M. G. A. Quantum estimation for quantum technology. Int. J. Quant. Inf. 7, 125 (2009).

45. Crowley, P. J. D., Datta, A., Barbieri, M. \& Walmsley, I. A. Multiparameter quantum metrology. arXiv:1206.0043 (2012).

46. Genoni, M. G. et al. Optimal estimation of joint parameters in phase space. arXiv:1206.4867 (2012)

47. Gill, R. D. \& Guta, M. On asymptotic quantum statistical inference. arXiv:1112.2078 (2011)

48. Lloyd, S. Universal quantum simulators. Science 273, 1073 (1996).

49. Aaronson, S. \& Arkhipov, A. The computational complexity of linear optics. arXiv:1011.3245 (2010).

50. Gruca, J., Laskowski, W. \& Zukowski, M. Nonclassicality of pure two-qutrit entangled states. Phy. Rev. A 85, 022118 (2012).

\section{Acknowledgements}

This work was supported by FIRB-Futuro in Ricerca HYTEQ, ERC-Starting Grant 3D-QUEST.

\section{Author contributions}

All authors contributed to design the ideas, perform the calculations, analyse the results and write the manuscript.

\section{Additional information}

Supplementary information accompanies this paper at http://www.nature.com/ scientificreports

Competing financial interests: The authors declare no competing financial interests.

License: This work is licensed under a Creative Commons

Attribution-NonCommercial-NoDerivs 3.0 Unported License. To view a copy of this license, visit http://creativecommons.org/licenses/by-nc-nd/3.0/

How to cite this article: Spagnolo, N. et al. Quantum interferometry with three-dimensional geometry. Sci. Rep. 2, 862; DOI:10.1038/srep00862 (2012). 